\title{
Impact of an antimicrobial stewardship program in the antimicrobial-resistant and prevalence of clostridium difficile infection and amount of antimicrobial consumed in cancer patients
}

\author{
Masoud Mardani \\ Shahid beheshti university of medical sciences, Tehran \\ Sara Abolghasemi \\ Shahid Beheshti University of Medical Sciences \\ Shiva Shabani ( $\nabla$ drshivashabani@gmail.com ) \\ Shahid Beheshti University of Medical Sciences
}

\section{Research note}

Keywords: ASP intervention, Clostridium difficile infection (CDI), Antimicrobial resistance, Meropenem, Vancomycin

Posted Date: November 21st, 2019

DOl: https://doi.org/10.21203/rs.2.17475/v1

License: (c) (1) This work is licensed under a Creative Commons Attribution 4.0 International License. Read Full License

Version of Record: A version of this preprint was published at BMC Research Notes on May 19th, 2020. See the published version at https://doi.org/10.1186/s13104-020-05085-3. 


\section{Abstract}

Objective: The objective of this study was to determine the impact of a hospital antimicrobial stewardship on antimicrobial resistant, clostridium difficile rates and amount of antimicrobial consumed in cancer patients. The intervention effects of ASP plans in 2017-2018 and 2018-2019 were respectively evaluated among hematology/oncology and bone morrow transplant patients in Ayatollah Taleghani University Hospital, Tehran, Iran. In this interventional quasi-experimental study, the ASP repository was utilized to capture four survey questions encompassed in these immunocompromised patients: amount of antibiotics (MPM and VMN) consumption gr-year, number of positive clostridium difficile infection and multidrug resistant positive cases in blood cultures.

Results: The number of MDR cases in the time periods of 2017-2018 and 2018-2019 were 145 and 75, respectively $(p=0.011)$. A significant reduction in positive blood cultures from 2017-2018 to 2018-2019 was found $(p=0.001)$. The amount of MPM prescriptions in 2018-2019 was significantly decreased from 22464 to $17262 \mathrm{~g} \mathrm{(}=0.043)$. The significant reduction in antibiotic consumption, MDR organisms and CDI can highly promote patients' health and decreasing medical costs and long-term defects for patients. Finally, future stewardship studies should made efforts for joining clinical and epidemiological studies.

\section{Introduction}

Antibiotic overuse and resistance problems have become a major public health issue worldwide (1). The inappropriate or overuse antibiotic usage, especially broad-spectrum agents results in the emergence and spread of antibiotic-resistant bacteria, which lead to increased morbidity, inpatient stays, and higher mortality $(1,2)$.

The problem of increasing antimicrobial resistance is especially critical for patients in the intensive care unit (ICU), because they are more susceptible to health care associated Infections (3).Increased multidrug resistance (MDR) organisms and Clostridium difficile infections have been involved in solid-organ transplant (SOT) recipients and have been associated with significant graft loss and mortality (4). Rates of bacterial infections in SOT recipients range from $21 \%$ to $68 \%$, depending on transplant type and immunotherapy (5). Between 20 to $40 \%$ patients receiving bone marrow transplant (would require ICU admission in the initial post transplantation Phase and the rates of their mortality was around $60 \%$ which have been reported for infectious diseases (6). Similarly, the incidence of Clostridium difficile infection $(\mathrm{CDI})$ in this population is increasing and characterized by more severe disease, frequent recurrences, and graft loss (4). There are limited treatment options for MDR organisms, and antibiotic exposure remains the principal risk factor for $\mathrm{CDI}$. Therefore, transplant providers, patient safety plan and programmatic interventions focusing on prevention are an emerging problem $(4,7)$.

Furthermore, the unrealized microbiome effects of altering immune function may predispose to allograft rejection. (4). Over the past few decades, published literature related to safety and efficacy of antibiotic 
stewardship strategies in high-risk immunocompromised hosts such as SOT recipients and those with hematologic malignancies exposed to cytotoxic chemotherapy and prolonged neutropenia is limited (8).

Multidrug resistance (MDR) is defined as resistant microorganism to the administered antimicrobial agents despite earlier sensitivity to it. These resistant microorganisms could rise in immunocompromised conditions, like individuals who have undergone organ transplantation leads further spread of MDR (9).

There has been a dramatic reduction in the development of antimicrobial agents with novel mechanisms of action to combat the rising spread of infections caused MDR organisms, although implementation challenges in immunocompromised hosts have been described $(4,8)$. In general, antimicrobialstewardship programs (ASPs) can help to improve clinical outcomes, limit antibiotic resistance and reduce medical costs by limiting the inappropriate use of antimicrobials in hospitals $(1,3,10)$.

It is unknown whether antimicrobial stewardship efforts are enclosing only the general patient population or whether immunocompromised hosts such as cancer and SOT are being included $(7,8)$. Duration of antimicrobial therapy and difficulty with source control is not clearly defined in many infections for immunocompromised hosts which may lead clinicians to prescribe broader and longer courses of antimicrobials than they would with a normal host $(5,8)$.

Among adult hospital ASPs, studies have indicated a reduction in the hospital length of stay in ICU, a decrease in C.difficile rates, and a development in antibiotic susceptibilities of common pathogens (10). Adherence to antibiotic guidelines varies among countries and institutions and the results of a recent comprehensive, antimicrobial stewardship program among adult hospital ASPs, studies have indicated a reduction in the hospital length of stay in ICU, a decrease in $C$. difficile rates, and a development in antibiotic susceptibilities of common pathogens $(3,11)$.The objective of this study was to determine the impact of a hospital ASP on antimicrobial resistant, clostridium difficile rates and amount of antimicrobial consumed meropenem (MPM) and vancomycin (VMN) in cancer patients.

\section{Materials And Methods}

\section{Setting and population}

We evaluated intervention effects of an antimicrobial stewardship (ASP) plans among hematology/oncology and bone morrow transplant patients in Ayatollah Taleghani University Hospital, in Tehran, Iran. All patients aged $>18$ years admitted through the medical wards. In this interventional quasiexperimental study, the ASP repository was utilized to capture four survey questions encompassed in these immunocompromised patients: amount of antibiotics (MPM and VMN) consumption gr-year, number of positive clostridium difficile infection and MDR positive cases in blood cultures were compared during two time periods, the first period was considered as the 12 months before the ASP implementation, while the second period was the first 12 months that the ASP was in place. Table 1 summarizes all detailed data for in this study. 
$C D I$ case definition. A patient was diagnosed with $\mathrm{CDI}$ when he or she had diarrhea (three or more unformed stool movements within $24 \mathrm{~h}$ ) and tested positive in an approach included an ELISA A+B kits (Abnova, Catalog Number KA3202) (12).

$M D R$. It was defined as the ability of organisms to resistance to the inhibitory effects of at least one or two most effective antibiotics (9).

\section{Data collection}

During the study period The ASP team inspected the primary instructions and applied usage arrangements of MPM and VMN according to the antimicrobial susceptibility patterns of clinical isolates. Our ASP team was including specialist in infectious diseases, cancer and clinical microbiology. They applied usage arrangements of antibiotics according to the antimicrobial susceptibility patterns of antibiotic-resistant infections.

Antimicrobial stewardship best practices guiding principles include the recommendations on multidrug resistance bacteria in bone marrow transplant recipients and the Urinary Tract Infection Guideline from the American Society were performed where applicable $(13,14)$.

The ASP repository was utilized to capture the data collected on each patient review including: antibiotic(s) prescribed, dose of antibiotic(s) and clinical data. Bacterial blood infection was assessed by checking their records to find the positive Gram staining results and detection of MDR bacteria (Figure 1). Consumption of each antimicrobial was expressed as the defined gram per year for VAN and MPM before and after the ASP. The costs of antimicrobial agents were not standardized, because they changed significantly during the study period. In addition, $C$. difficile specimens were evaluated using ELISA assay kit. Briefly, liquid stool specimens were screened for detection of toxin A and B by the clinical microbiology laboratory. CDI data were obtained at wards and summarized as all cases per year.

\section{Statistical analysis}

The data were statistically assessed using SPSS software package, version 21.0 (SPSS Inc., Chicago, IL, USA). The independent $t$-student test and Chi square $(\chi 2)$ or Fisher's exact tests were performed to assess between-group differences. The significant level was set at $p<0.05$.

\section{Results}

We collected the number and percent of fecal specimens in the various hospital wards of oncology and transplant populations. The absolute number of CDI was reduced from $11.2 \%$ patients per year in bone marrow transplantation wards in 2017- 2018 (period 2) to 2.7\% per year in 2018- 2019 (period 1) $(\mathrm{P}=0.004)$ (Table 1). 
The number of MDR cases in the time periods of 2017-2018 and 2018-2019 were 145 and 75, respectively ( $\mathrm{p}=$ 0.011). As shown in Table 2, a significant reduction in positive blood cultures from 2017-2018 to 2018-2019 was found $(\mathrm{p}=0.001)$.In addition, the amount of MPM prescriptions in 2018-2019 was significantly decreased from 22464 to $17262 \mathrm{~g}(\mathrm{p}=0.043)$.

Table 1. A summary of antibiotic prescriptions, MDR and CDI positive cases issued for the studied patients in time periods of 2017- 2018 and 2018-2019.

\begin{tabular}{|c|c|c|c|c|}
\hline Characteristics & \multicolumn{2}{|r|}{ 2018-2019 (without ASP) } & (with & $\begin{array}{l}\mathrm{P}- \\
\text { value }\end{array}$ \\
\hline \multicolumn{5}{|l|}{ Antibiotics (g) } \\
\hline $\mathrm{MPM}^{\mathrm{a}}$ & 22464 & 17262 & & 0.043 \\
\hline VMN & 15180 & 14400 & & 0.07 \\
\hline $\mathrm{MDR}^{\mathrm{a}}$ & 145 & 75 & & 0.011 \\
\hline \multicolumn{5}{|c|}{ CDI positive cases in wards ( $\mathrm{n} \%$ ) } \\
\hline $\begin{array}{l}\text { Bone marrow transplant } \\
\mathrm{a}, \mathrm{b}\end{array}$ & $5 / 43(11.6)$ & $1 / 37(2.7)$ & & 0.004 \\
\hline Oncology & $4 / 47(8.5)$ & $4 / 51(7.8)$ & & 0.09 \\
\hline
\end{tabular}

a Values in the same row followed by different letters $(\mathrm{a}, \mathrm{b})$ are significantly

b Bone marrow transplantation

Fig. 1 The admission number of positive and negative blood cultures in different wards of Ayatollah Taleghani Hospital during time periods of 2017-2018 and 2018-2019.

\section{Discussion}

It is worth recalling that the implementation of ASP guideline could promote the management of patients in different wards of hospitals. The development of evidence-based practice guidelines incorporating local microbiology and resistance patterns is strongly recommended in ASP $(11,15)$. This is a comprehensive approach to improve the pattern and amount of antibiotic prescriptions to reach the best clinical treatment response of the patients especially in immunocompromised patients $(8,11,16)$. 
The extensive application of ASPs in oncology and/or transplant patients have been associated with decreasing the inappropriate use of antimicrobial and cost reduction without resulting in harm in many countries $(7,17-19)$.

In a survey estimating ASPs at United States transplant centers, guideline development was a highly utilized antimicrobial stewardship intervention, occurring in 76 and $71 \%$ of hematopoietic stem cell transplant and solid organ transplant centers, respectively $(7,8)$. We couldn't assess the economic benefit of this culture-guided ASP, because the total cost of drugs were improved from 2017 to 2019. The results indicated that the new antimicrobial restriction policy reduced the treatment amount of MPM successfully $(P=0.043)$ in this hospital.

Transplant patients use the wide spectrum antibiotics and recent transplant cohort studies revealed significantly lower susceptibilities of common Gram-negative organisms isolated from their urine and blood (20). In this study, the outcomes of ASP method to control the antibiotic use VMN in healthcare settings was not improved $(P=0.07)$. This stewardship-discordant has been reported in another similar study that more than $40 \%$ of SOT patients were categorized as being discordant with stewardship principles. It was concluded from the uncertainty in the early stage of diagnosis $(5,21)$. Results of other extensive plans showed that the ASP utilization can significantly reduce the true antimicrobial drug with an appropriate antibiotic dose, and duration of MPM, VMN and diminish the number of positive cultures (22-24).

Findings of other similar studies in Singapore, Iran and US in hospitalized children and women showed that the proper prescription of carbapenems using the ASP was increased significantly, also proved that the ASP protocol can be successfully applied to promote quality of care of hospitalized patients $(22,25$, 26).

According to the results of the recently comprehensive studies in Iran, a relatively high infection rate of $C$. difficile was observed in patients with hospital acquired diarrhea in large teaching hospitals especially in cancer patients (27). Similar to results of other studies in different parts of the world such as Canada, US and Iran the rates of CDI and MDR positive cases are decreased significantly in our patients $(P=0.004$ \& $0.011)$ respectively. $(5,23,28)$. Our finding that enhanced antibiotic stewardship, including the restriction in the use of high risk broad-spectrum antibiotics, including fluoroquinolones, clindamycin, and cephalosporins was associated with a reduction in the number of CDI and MDR cases.

Use of ASP method leads to the improvement of patients' health and would result in a better change from intravenous to oral antibiotic use (23).

However, the pattern and route of antibiotic therapy were not considered in our study which may be a practical issue in further studies especially in the immunocompromised population. The aim of future ASPs should include the avoidance of high-risk antibiotics which is coupled with infection control, lower rates of resistance and increased guideline submission. 


\section{Conclusions}

This study compares the clinical impacts of the ASP with the conventional method and reports an antimicrobial restriction policy for MPM antibiotic. This significant reduction in antibiotic consumption, MDR organisms and CDI can highly promote patients' health and decreasing medical costs and long-term defects for patients. Finally, future stewardship studies should made efforts for joining clinical and epidemiological studies.

\section{Limitations}

The pattern and route of antibiotic therapy were not considered in our study which may be a practical issue in further studies especially in the immunocompromised population.

\section{Abbreviations}

ASP: antimicrobial stewardship program; MPM: meropenem; VMN: vancomycin; MDR: multidrug resistant; ICU: intensive care unit; SOT: solid-organ transplant; CDI: Clostridium difficile infection.

\section{Declarations}

\section{Ethics approval and consent to participate}

This study was approved by the human research ethics committee of Shahid Beheshti University of Medical Sciences, Tehran, Iran (the grant No. 9820), and it was carried out in accordance with the approved guidelines. Written consent informed was obtained from all participants.

\section{Consent to publication}

Not applicable.

\section{Availability of data and materials}

All data generated or analysed during this study are included in this published.

\section{Competing interests}

The authors declare that they have no competing interests.

\section{Funding}




\section{Authors' contributions}

A S and M M conceived and designed the study and co- ordinated the manuscript. Sh Sh executed data collection, performed the statistical analysis and prepared the draft of the manuscript. All authors read and approved the final manuscript.

\section{Acknowledgments}

We would like to thank the Research center for gastroenterology \& liver diseases. We would like to thank the Research center for gastroenterology \& liver diseases. We would like to thank Mrs Fatemeh Rouzbehani for her special corporation in this study.

\section{References}

1. Wu C-T, Chen C-L, Lee H-Y, Chang C-J, Liu P-Y, Li C-Y, et al. Decreased antimicrobial resistance and defined daily doses after implementation of a clinical culture-guided antimicrobial stewardship program in a local hospital. Journal of Microbiology, Immunology and Infection. 2017;50(6):846-56.

2. Wathne JS, Kleppe LKS, Harthug S, Blix HS, Nilsen RM, Charani E, et al. The effect of antibiotic stewardship interventions with stakeholder involvement in hospital settings: a multicentre, cluster randomized controlled intervention study. Antimicrobial Resistance \& Infection Control. 2018;7(1):109.

3. Chen I-L, Lee C-H, Su L-H, Wang Y-CL, Liu J-W. Effects of implementation of an online comprehensive antimicrobial-stewardship program in ICUs: a longitudinal study. Journal of microbiology, immunology and infection. 2018;51(1):55-63.

4. Hand J. Strategies for Antimicrobial Stewardship in Solid Organ Transplant Recipients. Infectious disease clinics of North America. 2018;32(3):535-50.

5. So M, Yang DY, Bell C, Humar A, Morris A, Husain S. Solid organ transplant patients: are there opportunities for antimicrobial stewardship? Clinical transplantation. 2016;30(6):659-68.

6. Khan N and Rahman B. Infections After Bone Marrow Transplantation in Children - A Retrospective Analysis. Cientific Journal of Paediatrics. 2019;1(4):5-8.

7. Robilotti E, Holubar M, Seo SK, Deresinski S. Feasibility and applicability of antimicrobial stewardship in immunocompromised patients. Current opinion in infectious diseases. 
2017;30(4):346-53.

8. Seo SK, Lo K, Abbo LM. Current state of antimicrobial stewardship at solid organ and hematopoietic cell transplant centers in the United States. infection control \& hospital epidemiology. 2016;37(10):1195-200.

9. Tanwar J, Das S, Fatima Z, Hameed S. Multidrug resistance: an emerging crisis. Interdisciplinary perspectives on infectious diseases. 2014.

10. Lee BR, Goldman JL, Yu D, Myers AL, Stach LM, Hedican E, et al. Clinical Impact of an Antibiotic Stewardship Program at a Children's Hospital. Infectious Diseases Therapy. 2017; 6(1):103-3.

11. Wenisch JM, Equiluz-Bruck S, Fudel M, Reiter I, Schmid A, Singer E, et al. Decreasing Clostridium difficile infections by an antimicrobial stewardship program that reduces moxifloxacin use. Antimicrobial agents and chemotherapy. 2014;58(9):5079-83.

12. Patton A, Davey P, Harbarth S, Nathwani D, Sneddon J, Marwick CA. Impact of antimicrobial stewardship interventions on Clostridium difficile infection and clinical outcomes: segmented regression analyses. Journal of Antimicrobial Chemotherapy. 2017;73(2):517-26.

13. Yanai M, Ogasawara M, Hayashi Y, Suzuki K, Takahashi H, Satomura A.Impact of interventions by an antimicrobial stewardship program team on appropriate antimicrobial therapy in patients with bacteremic urinary tract infection. Journal of Infection Chemotherapy. 2018;24(3):206-11.

14. Dellit TH, Owens RC, McGowan JE, Jr, Gerding DN, Weinstein RA, Burke JP, Huskins WC, Paterson DL, Fishman NO, Carpenter CF, Brennan PJ, Billeter M, Hooton TM. Infectious Diseases Society of America and the Society for Healthcare Epidemiology of America guidelines for developing an institutional program to enhance antimicrobial stewardship. Clinical Infectious Diseases. 2007;15:44(2):159-77.

15. MacDougall C, Polk RE. Antimicrobial stewardship programs in health care systems. Clinical microbiology reviews. 2005;18(4):638-56.

16. Aitken SL, Palmer HR, Topal JE, Gabardi S, Tichy E. Call for antimicrobial stewardship in solid organ transplantation. American Journal of Transplantation. 2013;13(9):2499. 
17. Muraki Y, Kitamura M, Maeda Y, Kitahara T, Mori T, Ikeue H, et al. Nationwide surveillance of antimicrobial consumption and resistance to Pseudomonas aeruginosa isolates at 203 Japanese hospitals in 2010. Infection. 2013;41(2):415-23.

18. Storey DF, Pate PG, Nguyen AT, Chang F. Implementation of an antimicrobial stewardship program on the medical-surgical service of a 100-bed community hospital. Antimicrobial resistance and infection control. 2012;1(1):32.

19. Lin Y-S, Lin I-F, Yen Y-F, Lin P-C, Shiu Y-C, Hu H-Y, et al. Impact of an antimicrobial stewardship program with multidisciplinary cooperation in a community public teaching hospital in Taiwan. American journal of infection control. 2013;41(11):1069-72.

20. Rosa R, Simkins J, Camargo JF, Martinez O, Abbo LM. Solid organ transplant antibiograms: an opportunity for antimicrobial stewardship. Diagnostic microbiology and infectious disease. 2016;86(4):460-3.

21. Weston G, Jacob JT, Ray S, Varkey J, Gaynes RP. A multicenter study measuring appropriateness of carbapenem use. Infection Control \& Hospital Epidemiology. 2013;34(12):1324-6.

22. Cosgrove SE, Carmeli Y. The impact of antimicrobial resistance on health and economic outcomes. Clinical Infectious Diseases. 2003;36(11):1433-7.

23. Rahbarimanesh A, Mojtahedi SY, Sadeghi P, Ghodsi M, Kianfar S, Khedmat L, et al. Antimicrobial stewardship program (ASP): an effective implementing technique for the therapy efficiency of meropenem and vancomycin antibiotics in Iranian pediatric patients. Annals of clinical microbiology and antimicrobials. 2019;18(1):6.

24. Talbot GH, Bradley J, Edwards Jr JE, Gilbert D, Scheld M, Bartlett JG. Bad bugs need drugs: an update on the development pipeline from the Antimicrobial Availability Task Force of the Infectious Diseases Society of America. Clinical infectious diseases. 2006;42(5):657-68.

25. Seah XFV, Ong YLR, Tan SW, Krishnaswamy G, Chong CY, Tan NWH, et al. Impact of an antimicrobial stewardship program on the use of carbapenems in a tertiary women's and children's hospital, Singapore. Pharmacotherapy: The Journal of Human Pharmacology and Drug Therapy. 2014;34(11):1141-50.

26. Lee KR, Bagga B, Arnold SR. Reduction of broad-spectrum antimicrobial use in a tertiary children's hospital post antimicrobial stewardship program guideline implementation. Pediatric Critical Care Medicine. 2016;17(3):187-93.

27. Shoaei P, Shojaei H, Khorvash F, Ataei B, Esfandiari Z, Vakili B, et al.Clostridium difficile infection in cancer patients with hospital acquired diarrhea at the teaching hospitals in Iran: Multilocus sequence 
typing analysis (MLST) and Antimicrobial resistance pattern. Annali di igiene: medicina preventiva e di comunita. 2019;31(4):365-73.

28. Christensen AB, Barr VO, Martin DW, Anderson MM, Gibson AK, Hoff BM, et al. Diagnostic stewardship of C. difficile testing: a quasi-experimental antimicrobial stewardship study. Infection Control \& Hospital Epidemiology. 2019;40(3):269-75.

\section{Figures}

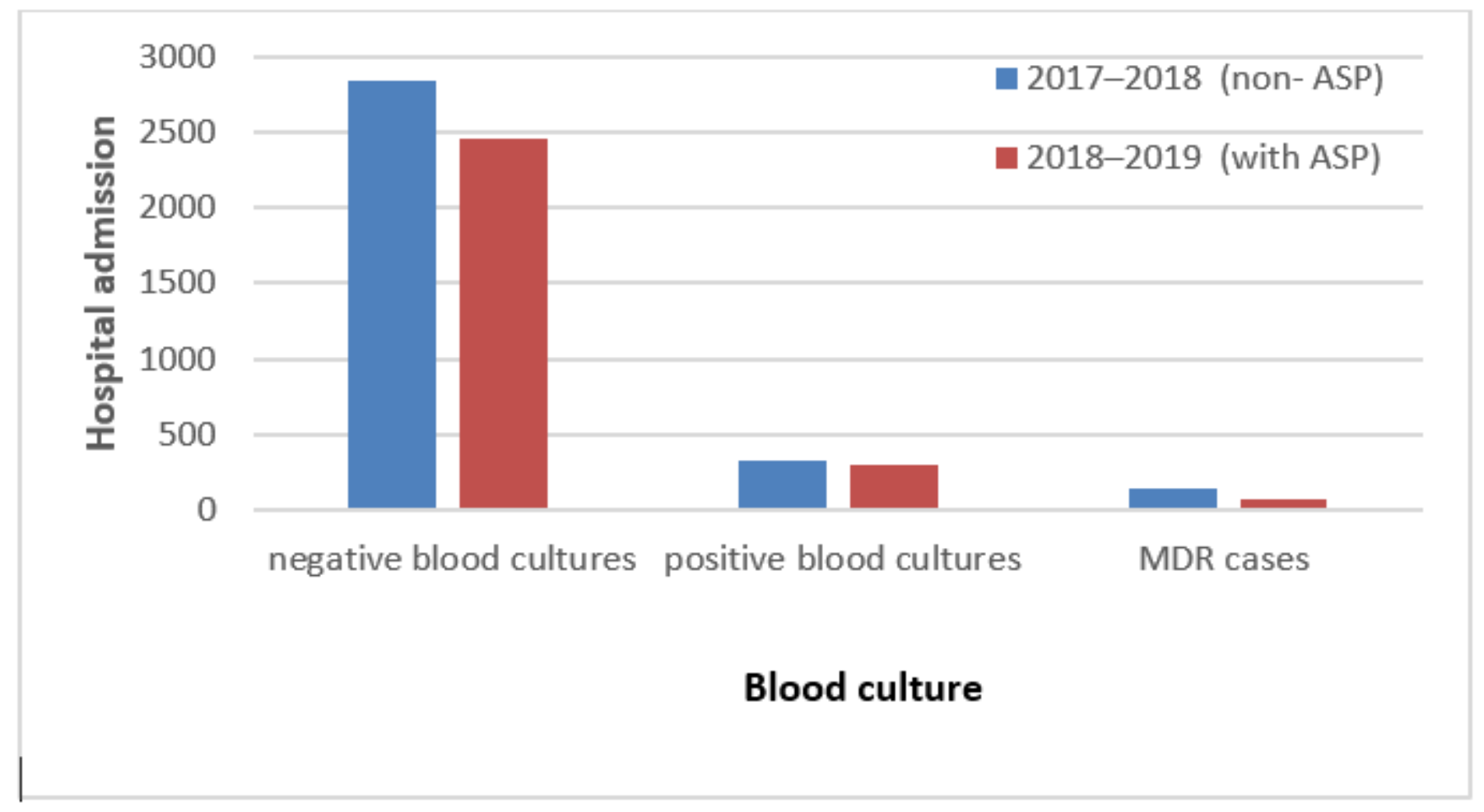

\section{Figure 1}

The admission number of positive and negative blood cultures in different wards of Ayatollah Taleghani Hospital during time periods of 2017-2018 and 2018-2019. 